\title{
Improvement of Care in Patients With Colorectal Cancer: Influence of the Introduction of Standardized Structured Reporting for Pathology
}

Caro E. Sluijter, MSc ${ }^{1,2}$; Frans van Workum, MD ${ }^{1}$; Theo Wiggers, MD, PhD ${ }^{3}$; Carlijn van de Water ${ }^{1}$; Otto Visser, MD, PhD ${ }^{4}$; Henk-Jan van Slooten, MD, PhD ${ }^{2,5}$; Lucy I.H. Overbeek, PhD²; and Iris D. Nagtegaal, MD, PhD ${ }^{1,2}$

PURPOSE The use of standardized structured reporting (SSR) can improve communication between cancer specialists, which might improve clinical care; however, there are no reliable data on whether the introduction of SSR is associated with improvements in clinical outcome.

PATIENTS AND METHODS We performed a retrospective cohort study in the Netherlands, including all patients with colorectal cancer (CRC) from 2009 to 2014. As a reference, cohorts of 2007 and 2008 were included. Data from the Netherlands Cancer Registry were used and combined with data from the Dutch Pathology Registry (PALGA) and the Dutch ColoRectal Audit. We tested the preformulated hypothesis that use of SSR improves the care of patients with CRC by improving the completeness of the pathology reports, the quality of the pathology evaluation, and patient outcomes with respect to treatment and survival.

RESULTS We included 72,859 patients with CRC (23.8\% reference, 32.9\% SSR, and $43.3 \%$ narrative reports). Use of SSR increased over time, which resulted in more complete pathology reports (95.8\% v89.8\%; $P<.001)$. Risk assessment in stage II colon cancer was more adequate and resulted in an increased delivery of adjuvant therapy in patients with SSR $(19.6 \% \vee 15.1 \% ; P=.001)$. Risk of death for patients in the SSR group was significantly lowered (corrected hazard ratio, $0.94 ; 95 \% \mathrm{Cl} 0.90$ to 0.97 ).

CONCLUSION We demonstrate that use of SSR improved patient care in those with CRC by providing more complete reports of higher quality, which had significant effects on the delivery of adjuvant therapy and patient outcomes.

Clin Cancer Inform. @ 2019 by American Society of Clinical Oncology

\section{INTRODUCTION}

With the increasing complexity of modern multidisciplinary cancer treatment, adequate exchange of diagnostic information is increasingly important. Postoperative pathology reports describe the definitive staging and typing of tumors and are the basis of additional treatment plans. For colorectal cancer (CRC), the decision of whether to administer adjuvant chemotherapy is dependent on tumor stage and high-risk features. It has been demonstrated that adjuvant chemotherapy results in a survival benefit for all stage III patients and those with high-risk stage II disease. ${ }^{1}$ Highrisk features of stage II CRC_-poor differentiation, extramural vascular invasion (EMVI), tumor perforation, T4, fewer than 10 examined lymph nodes, and obstruction/ileus - are mainly determined by the pathologist. Therefore, the accuracy and completeness of pathology reports are important for adequate adjuvant chemotherapy delivery and patient outcome. ${ }^{2}$

Traditional narrative reports (NRs) are no longer considered adequate as it can be difficult to distill the necessary information from long-winded sentences that describe morphologic features rather than clinically relevant information. In standardized structured reporting (SSR) systems, mandatory parameters are defined and presented in a standardized structure, thereby improving informational content ${ }^{3}$. Use of these systems has been shown to improve both the readability and completeness of postoperative pathology reports. ${ }^{4}$ Several smaller studies have proven that use of SSR resulted in increased completeness for a variety of prognostic factors, such as involvement of the circumferential resection margin (CRM), ${ }^{5-11}$ EMVI, $5,9,11,12$ and TNM stage. ${ }^{7,10,11,13}$ However, whether this also leads to increased quality of the diagnostic process and subsequent treatment decisions has not yet been explored.

In the Netherlands, we introduced SSR nationwide for CRC resections in 2009. All mandatory parameters were defined in the Dutch guidelines for the diagnosis and treatment of CRC. ${ }^{14}$ To explore the impact of SSR on the diagnosis and treatment of patients with CRC and to test the hypothesis that the introduction of SSR 


\section{CONTEXT}

\section{Key Objective}

To investigate the impact of standardized synoptic pathology reporting on the diagnosis and treatment of patients with colorectal cancer in a nationwide cohort study.

\section{Knowledge Generated}

Implementation of standardized structured reporting of pathology findings for patients with colorectal cancer resulted in increased completeness of pathology reports and higher-quality pathology evaluation, and was associated with better outcome for patients.

\section{Relevance}

These findings support the widespread introduction of standardized structured pathology reporting in oncology.

was associated with increased completeness of reporting, increased reporting of quality indicators, and improved patient outcomes, we performed a population-based retrospective cohort study.

\section{PATIENTS AND METHODS}

\section{Design, Data Sources, and Population}

We conducted a population-based retrospective nationwide cohort study in which all surgically treated patients with primary CRC diagnosed between 2007 and 2014 from the Netherlands Cancer Registry (NCR) were included..$^{15}$ Data from the NCR were used as all data were retrieved from both the NR and SSR for this registration. NR and SSR were compared for all outcome measures from the introduction of SSR in 2009 until 2014. To observe ongoing trends independent of the introduction of SSR, we used cohorts from 2007 to 2008 as a reference group. Linkage of the NCR with the Dutch Pathology Registry-PALGA ${ }^{16}$ -and the Dutch ColoRectal Audit (DCRA; formerly the Dutch Surgical Colorectal Audit) ${ }^{17}$ was performed. PALGA is the nationwide network and registry of histo- and cytopathology in the Netherlands, with nationwide coverage since 1991. The DCRA is part of the Dutch Institute for Clinical Auditing and registers all patients who undergo surgery for CRC since 2009.

Patients were selected using International Classification of Diseases, Oncology codes (3rd edition ${ }^{18}$ ) C18.0 to C18.9 (colon, excluding appendix C18.1), C19.9 (rectosigmoid), and C20.9 (rectum). Rectosigmoid tumors were classified as either colon or rectum on the basis of the conclusions of the pathology reports. Patients with CRC who did not undergo resection, patients with multiple synchronous CRC or in situ carcinomas, and resection specimens without tumor were excluded, as well as those CRC cases that were reported with SSR before 2009 (minor pilot project, including 243 patients).

Variables retrieved from the NCR were as follows: gender, age at diagnosis, follow-up time, vital status, year of diagnosis, localization, histologic type, histologic grade, invasion depth ( $\mathrm{pT}$ ), nodal status ( $\mathrm{pN}$ ), presence of metastases
(pM), number of investigated and positive lymph nodes (LNs), CRM, and primary treatment of CRC. Three high-risk features, including EMVI data, obstruction, and bowel perforation were retrieved from the DCRA. Type of report (SSR or NR), date of resection, type of pathology laboratory (academic/university associated $v$ nonacademic/nonuniversity associated), and random laboratory number were retrieved from PALGA.

The Dutch SSR is considered level six on the Ontario Scale. ${ }^{3}$

\section{Completeness, Quality Indicators, and Patient Outcomes}

We assessed the completeness of pathology reports using two different definitions: first, overall completeness-that is, the proportion of pathology reports that contained all mandatory pathology parameters-and second, parameter-specific completeness-that is, the proportion of pathology reports in which an individual parameter was reported. Parameters were selected according to the Dutch guideline ${ }^{14}$ in combination with availability in the NCR and DSCA. Six mandatory parameters-histologic type, histologic grade, pT, pN, LN count, and CRM — and one optional parameter-EMVI-were analyzed. Histologic grade was analyzed for patients who did not receive neoadjuvant therapy, and CRM was analyzed for patients with rectal resections only. As the NCR began registrations of CRM in 2008, we could not perform this analysis in the 2007 cohort reference group. EMVI was analyzed for patients who were diagnosed in 2009 to 2014, because the registration of this parameter by DCRA began in 2009. The other four parameters were analyzed for the whole study population.

The quality of pathology evaluation was defined as the proportion of pathology reports in which the informational content corresponded to Dutch and international quality standards. We examined the number of $\mathrm{LNs},{ }^{14}$ reporting of EMVI, ${ }^{19}$ and CRM. In addition, we used the prognostic power of these histologic factors as a surrogate marker for quality.

To evaluate the effects of SSR on patient care, we analyzed the proportion of patients with high-risk stage II colon cancer who received adjuvant chemotherapy. In addition, 
we analyzed the effect of SSR on patient survival. Survival time was defined as the time from diagnosis until death or last follow-up date (January 31, 2016).

\section{Statistical Analysis}

Statistical analyses were performed using SAS Enterprise Guide 7 (SAS/STAT User's Guide; SAS Institute, Cary, NC), and all statistical tests were two sided. We used basic descriptive statistics to describe patient and tumor characteristics for NR, SSR, and the reference group. Annual and overall proportions were calculated for overall completeness, completeness of specific parameters, and quality indicators for NR, SSR, the reference group, and the total study population. We used univariable and multivariable logistic regression analyses to describe the association between the type of reporting and outcome measures using crude and adjusted odds ratios (OR) with corresponding $95 \%$ Cls. Year of diagnosis was selected as an a priori confounding factor and included in every model.

To investigate the impact of the implementation of SSR, we plotted relevant outcome parameters against the consecutive case numbers before and after the introduction of SSR. To allow for the interpretation of whether results were based on casemix, we also plotted predicted values that were based on the casemix parameters stated above. Per hospital, cases before the introduction of SSR received negative consecutive case numbers and case 0 represents the first case in which an SSR was used. Data from all hospitals were pooled and we performed exponentially weighted-moving average analysis to analyze changing trends in outcome parameters that could be explained by the implementation of SSR. ${ }^{20}$

Proportions of patients with high-, low-, and undeterminedrisk stage II colon cancer and the corresponding proportions of patients who received adjuvant chemotherapy were calculated for SSR and NR and analyzed using $\chi^{2}$ analyses and adjusted ORs. We used Kaplan-Meier curves and log-rank tests to evaluate overall survival, stratified by the type of reporting. Survival was also calculated for the following quality indicators: EMVI (detected, not detected, or missing), CRM (negative, positive, or missing), and number of LNs (more or less than 10 or missing). The prognostic value of quality indicators in NR and SSR was assessed using the Akaike information content (AIC) ${ }^{21} \mathrm{Cox}$ proportional hazards analyses were used to obtain hazard ratios (HR) and corresponding 95\% $\mathrm{Cls}$. Associations were adjusted in multivariable analyses for age at diagnosis, gender, year of diagnosis, localization of tumor (not for CRM analysis), histologic type, histologic grade, TNM stage, and type of pathology laboratory. We tested the proportional hazards assumption using Schoenfield residuals and found it to be satisfied.

\section{RESULTS}

The initial selection from the NCR resulted in 109,560 primary CRCs from 105,720 patients who were diagnosed between 2007 and 2014 (Fig 1). After linkage with PALGA ( $n=104,925 ; 95.8 \%$ ) and exclusion of tumors of origin other than colon or rectum $(n=16)$, in situ tumors ( $n=$ 4,044), CRC without surgical intervention ( $n=20,676$ ), pT0 and pTx resections $(n=2,228)$, synchronous CRC $(n=$ 3,913), and SSR from 2007 or $2008(n=243)$, a total of 73,805 CRCs that occurred in 72,859 patients were included for analysis. Table 1 lists patient and tumor characteristics of the study population. There were 17,489 pathology reports in the reference group (23.7\%), 32,065 NR (43.4\%), and 24,251 SSR (32.9\%). Over time, use of SSR increased from $11.1 \%$ in 2009 to $78.3 \%$ in 2014 (Fig 2A). In the teaching setting of academic pathology laboratories, pathologists used NR more frequent than SSR, as was the case in stage IV CRC, rectal cancer, and those patients who were treated with neoadjuvant therapy. SSR was used more frequently than NR for low-grade tumors as well as for more adenocarcinomas, not otherwise specified.

\section{Completeness}

In the reference group, $90.5 \%$ of reports were already complete. As a result of the introduction of SSR, completeness increased (Fig 3A), whereas NR remained on the level of the reference group (95.8\% v89.8\%; OR, 2.6; 95\%

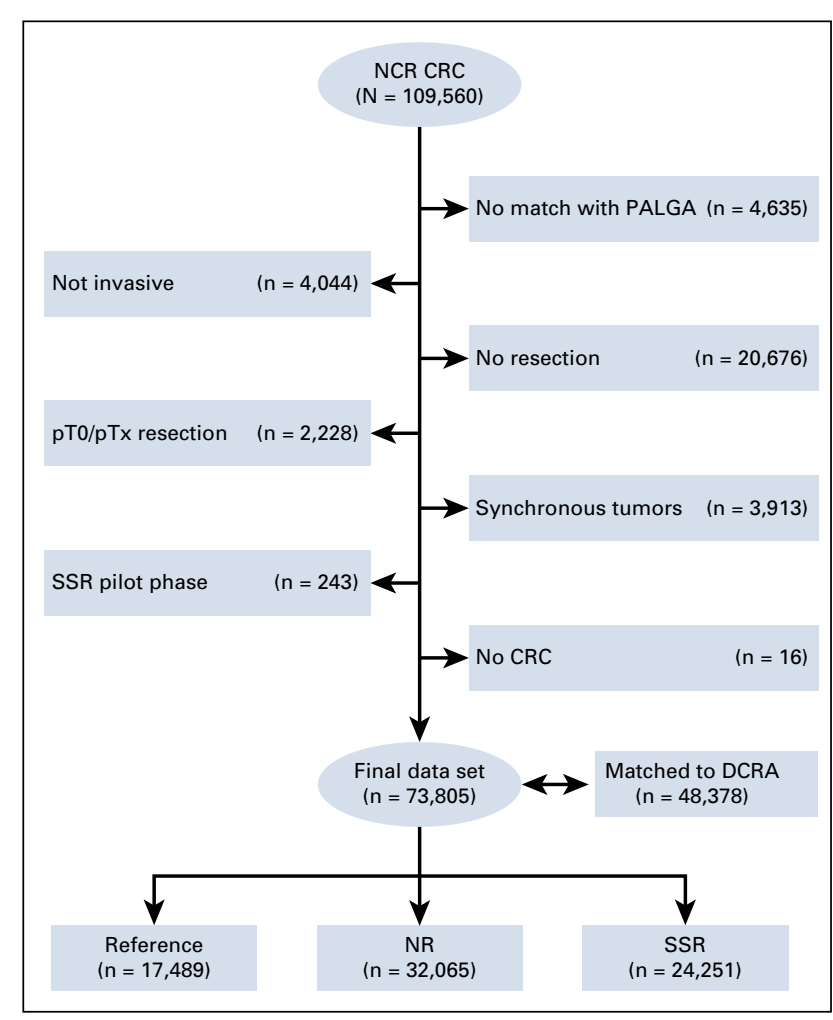

FIG 1. Flowchart of the patients with colorectal cancers (CRCs) who were included in the study. DCRA, Dutch ColoRectal Audit; NCR, Netherlands Cancer Registry; NR, narrative report; PALGA, Dutch Pathology Registry; SSR, standardized structured reporting. 
TABLE 1. Patient and Tumor Characteristics

Characteristic

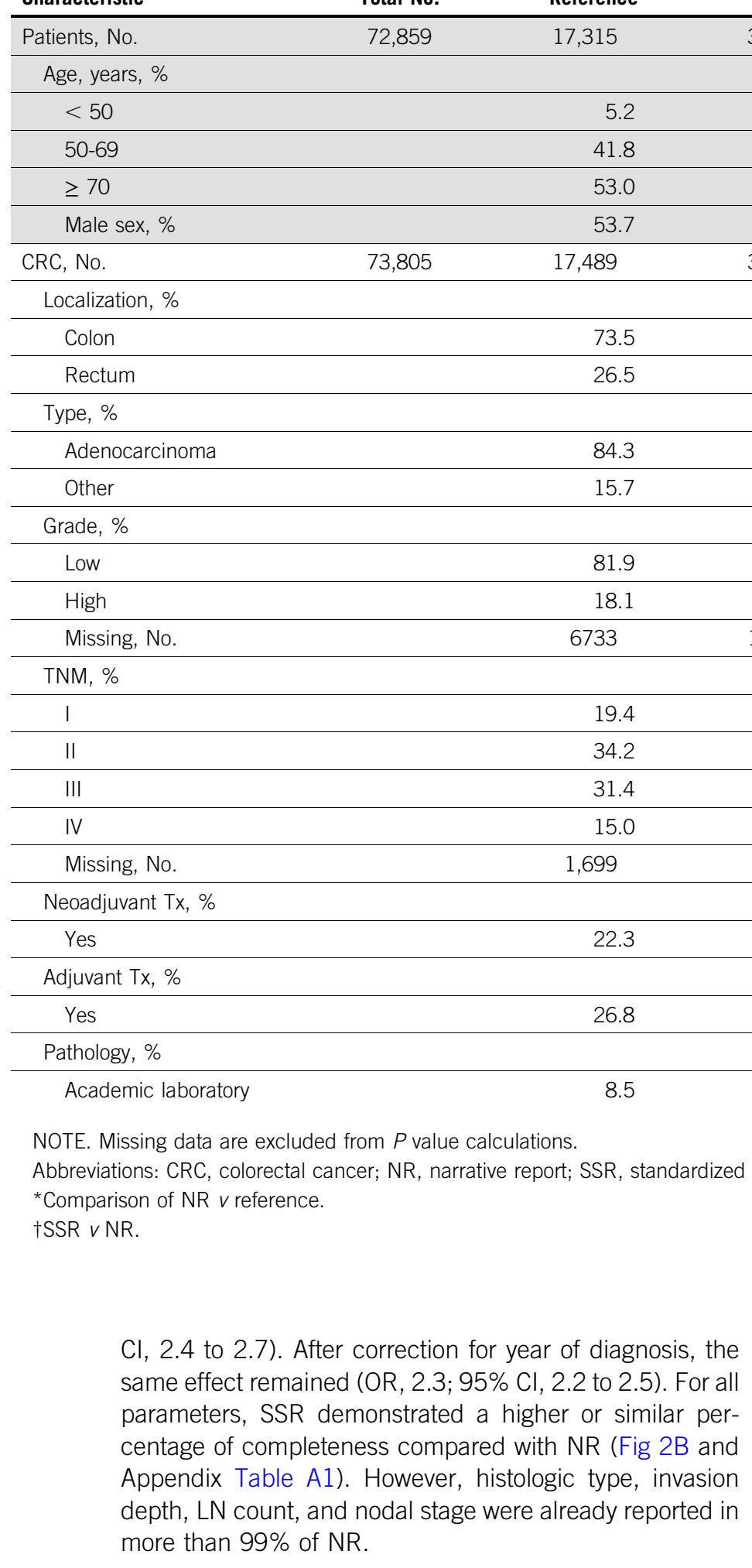

\section{Quality Indicators}

Introduction of SSR was associated with a remarkable increase in the number of investigated LNs (Fig 3B). As a consequence, there was a significant increase in the number of cases in which more than 10 LNs were

NOTE. Missing data are excluded from $P$ value calculations.

${ }^{*}$ Comparison of NR $v$ reference.
$\mathrm{Cl}, 2.4$ to 2.7). After correction for year of diagnosis, the parameters, SSR demonstrated a higher or similar percentage of completeness compared with NR (Fig 2B and depth, LN count, and nodal stage were already reported in more than $99 \%$ of NR.
NR

SSR

31,566

23,978

5.6

43.3

4.4

.002

$<.001$

51.1

41.5

54.9

54.1

54.3

-

24,251

32,065

69.5

79.1

30.5

20.9

$85.6 \quad 88.6$

11.4

14.4

83.7

16.3

88.2

14,579

11.8

7,674

\begin{tabular}{cccc}
22.3 & 21.6 & $<.001$ & $<.001$ \\
33.4 & 34.5 & - & - \\
30.8 & 32.7 & - & - \\
13.5 & 11.2 & - & - \\
838 & 142 & - & - \\
\hline
\end{tabular}

\begin{tabular}{cccc}
22.3 & 21.6 & $<.001$ & $<.001$ \\
33.4 & 34.5 & - & - \\
30.8 & 32.7 & - & - \\
13.5 & 11.2 & - & - \\
838 & 142 & - & - \\
\hline
\end{tabular}

28

28.1

14.6

$<.001$

$<.001$

25

25.2

26.4

$<.001$

$<.001$

12.0

5.7

$<.001$

$<.001$

\begin{tabular}{lll} 
& & \\
26.4 & $<.001$ & $<.001$ \\
\hline 5.7 & $<.001$ & $<.001$
\end{tabular}

investigated (SSR, 89.3\% v NR, 77.3\%; OR, 1.8; 95\% Cl, 1.7 to 1.9 , adjusted for year of diagnosis; Fig 2C). There was a small difference in the detection of EMVI (SSR, $17.1 \% \vee N R, 16.1 \% ; P=.003$ ). Although the reporting of the CRM increased over time (Fig 2D), there was no difference in the percentage of CRM positivity. To determine the adequacy of the histologic features reported, we compared the prognostic value of those features in the different settings applying AIC statistics. The prognostic model was better in the SSR cases for EMVI (AIC, 92,966 v 169,371; Appendix Figs A1, A2), CRM involvement (AIC, 12,322 v 46,956; Appendix Figs A3, A4), and number of LNs (AIC, 80,397 v 1,123,778; Appendix Figs A5, A6). 


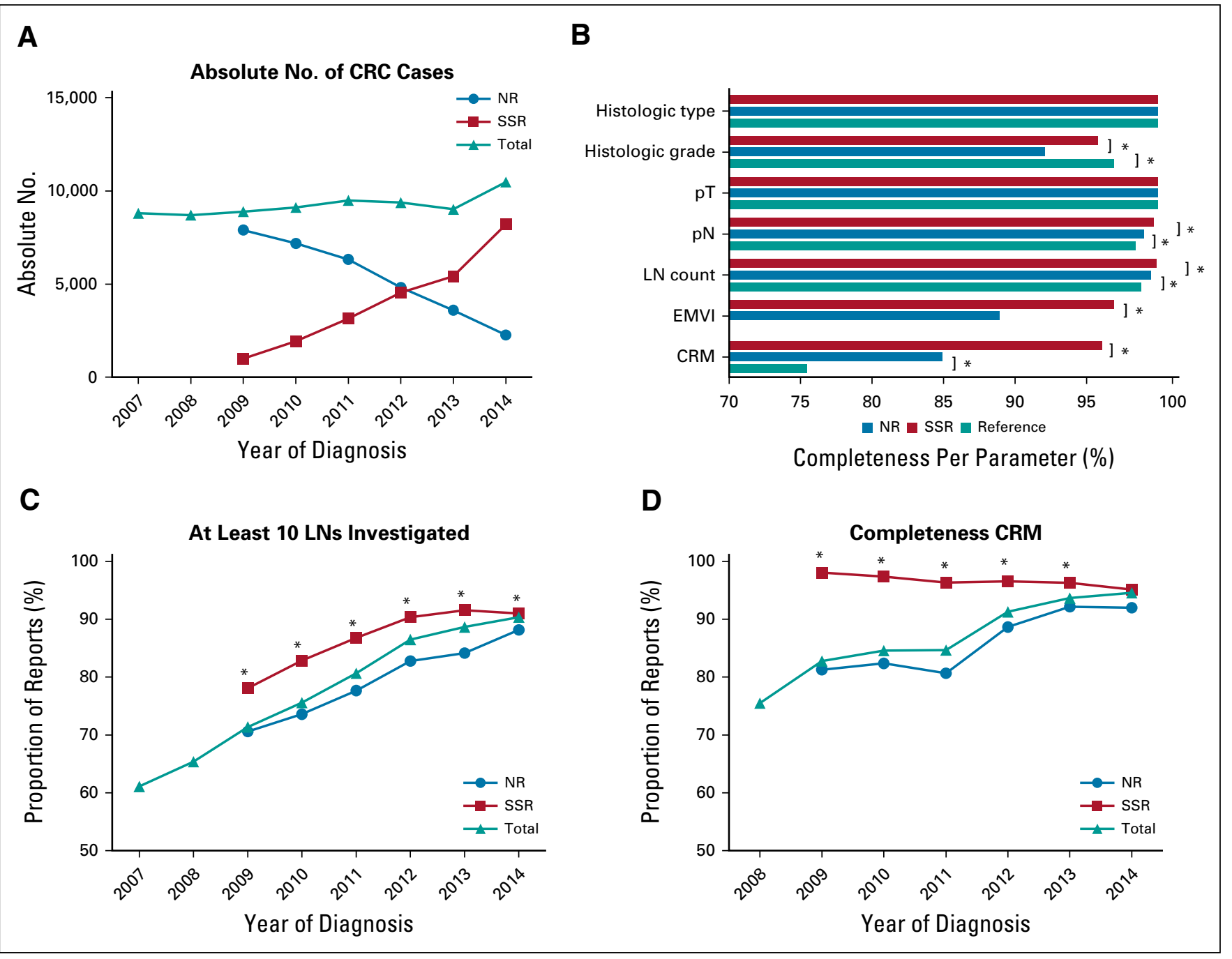

FIG 2. Effects of the introduction of standardized structured reporting (SSR) in the Netherlands. (A) Introduction of SSR in the Netherlands compared with the use of narrative report (NR). (B) Completeness per factor; NR and SSR are compared with the reference cohort. (C) Increased number of cases with more than 10 lymph nodes (LNs) examined over time; total numbers are compared with NR and SSR. (D) Completeness of circumferential resection margin (CRM) reporting over time; total numbers are compared with NR and SSR. CRC, colorectal cancer; EMVI, extramural vascular invasion. ${ }^{*} P<.001$.

\section{Impact on Patient Care and Outcome}

The data set consisted of 12,366 patients with stage II colon cancer who were diagnosed between 2009 and 2014 and who were potentially eligible for adjuvant therapy. Of these, 7,202 patients were considered low risk (SSR, 64.2\% vNR, 52.7\%; $P<.001$ ) and 4,381 patients were high risk (SSR, $31.2 \%$ v NR, 39.4\%; $P<.001$ ). Risk status could not be determined for 783 patients as a result of missing information on one or more risk factors (SSR, $4.6 \% v \mathrm{NR}$, $8.0 \% ; P<.001$ ). Of high-risk patients with SSR, $19.6 \%$ received chemotherapy compared with $15.1 \%$ of high-risk patients with NR $(P=.001)$, even when corrected for year of diagnosis, gender, and age (adjusted OR, 1.29; $95 \% \mathrm{Cl}$, 1.06 to 1.57). No difference in chemotherapy administration was observed for low-risk patients, nor for patients with an unknown risk status. Overall survival in the high-risk group was significantly better in patients with SSR $(65.0 \% \mathrm{~V}$
$62.4 \% ; P=.026$; Fig 4A). No differences were observed for patients with low or unknown risk in relation to the type of report.

Crude 5-year overall survival for patients with an SSR was higher compared with patients with an NR $(64.9 \% \quad v$ 62.2\%; $P<.001$; Fig 4B). As there were significant differences in stage, grade, and neoadjuvant therapy between SSR and NR (Table 1), we adjusted for these factors, as well as for age at diagnosis, gender, year of diagnosis, localization of tumor, histologic type, and type of pathology laboratory. The difference remained present after adjustment (HR, 0.94; $95 \% \mathrm{Cl}, 0.90$ to 0.97 ).

\section{DISCUSSION}

We demonstrated that the nationwide implementation of SSR for CRC resection specimens resulted in improved completeness and quality of pathology reports, and we 

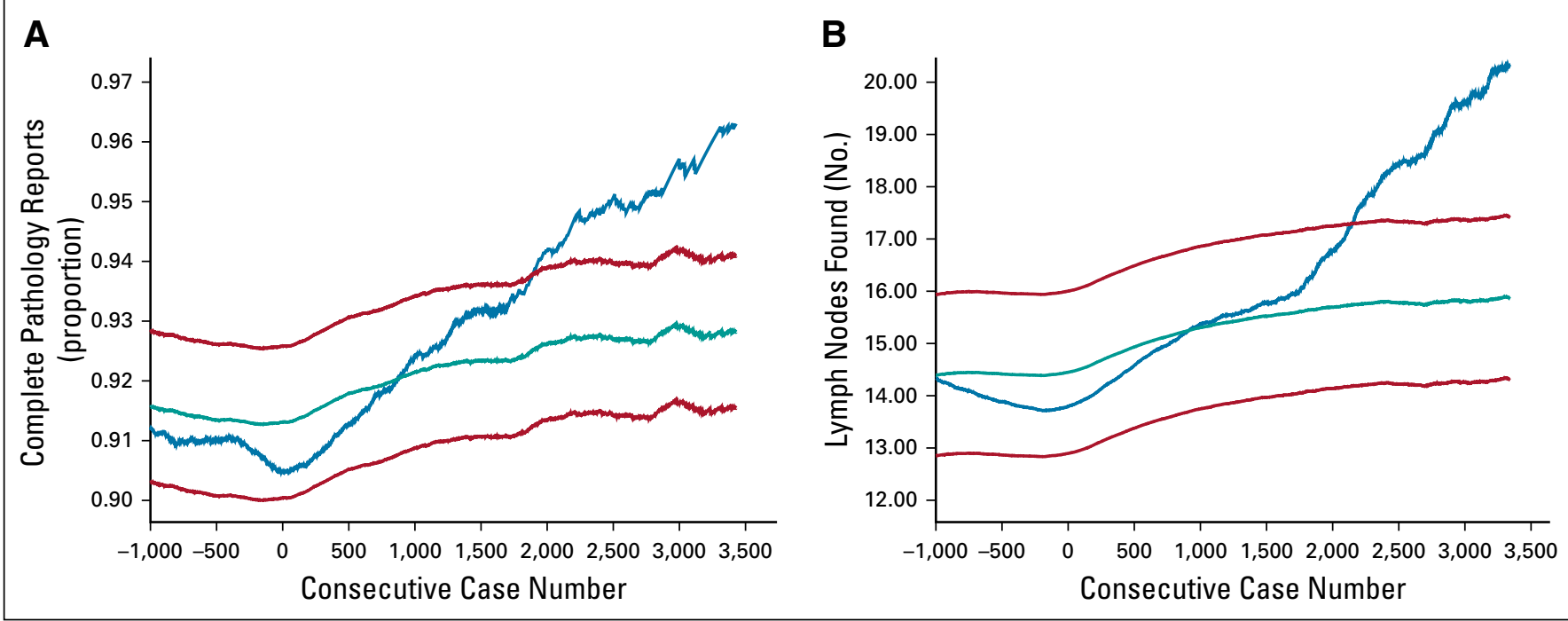

FIG 3. (A) Proportion of complete pathology reports after the introduction of standardized structured reporting (SSR). Blue, observed proportion of complete reports. Teal, expected proportion of complete reports. Red, 95\% Cl for the predicted proportion of complete reports. SSR is introduced at consecutive case number 0 . After the observed proportion of complete reports exceeds the upper limit of the $95 \% \mathrm{Cl}$ of the expected proportion of complete reports, the increase of completeness can no longer be explained by casemix and is likely caused by the introduction of SSR. (B) Number of lymph nodes after the introduction of SSR. Blue, observed numbers of lymph nodes. Teal, expected number of lymph nodes. Red, $95 \% \mathrm{Cl}$ for the predicted number of lymph nodes. SSR is introduced at consecutive case number 0 . After the observed number of lymph nodes found exceeds the upper limit of the $95 \% \mathrm{Cl}$ of the expected number of lymph nodes found, the increase can no longer be explained by casemix and is likely caused by the introduction of SSR.

have described the effects on patient care. Improved completeness was expected on the basis of previous studies $^{4}$; however, these studies were relatively small, with only one study that included more than 1,000 SSR. Completeness for individual parameters was highly variable, but well below the $60 \%$ as a starting point for many parameters, such as resection margins ${ }^{6,12,22}$ and stage. ${ }^{10,11,13}$ Our reference group presented with more than $90 \%$ total completeness; therefore, we expected a limited effect of SSR on completeness. This potentially limited the impact of the introduction on health care improvement; however, we demonstrated that SSR further improves reporting of all relevant items to $95 \%$ to $100 \%$, which is in line with other publications. . $^{9,10,23,24}$ The improvement is particularly pronounced for more recently recognized parameters, such as EMVI and CRM. ${ }^{4}$
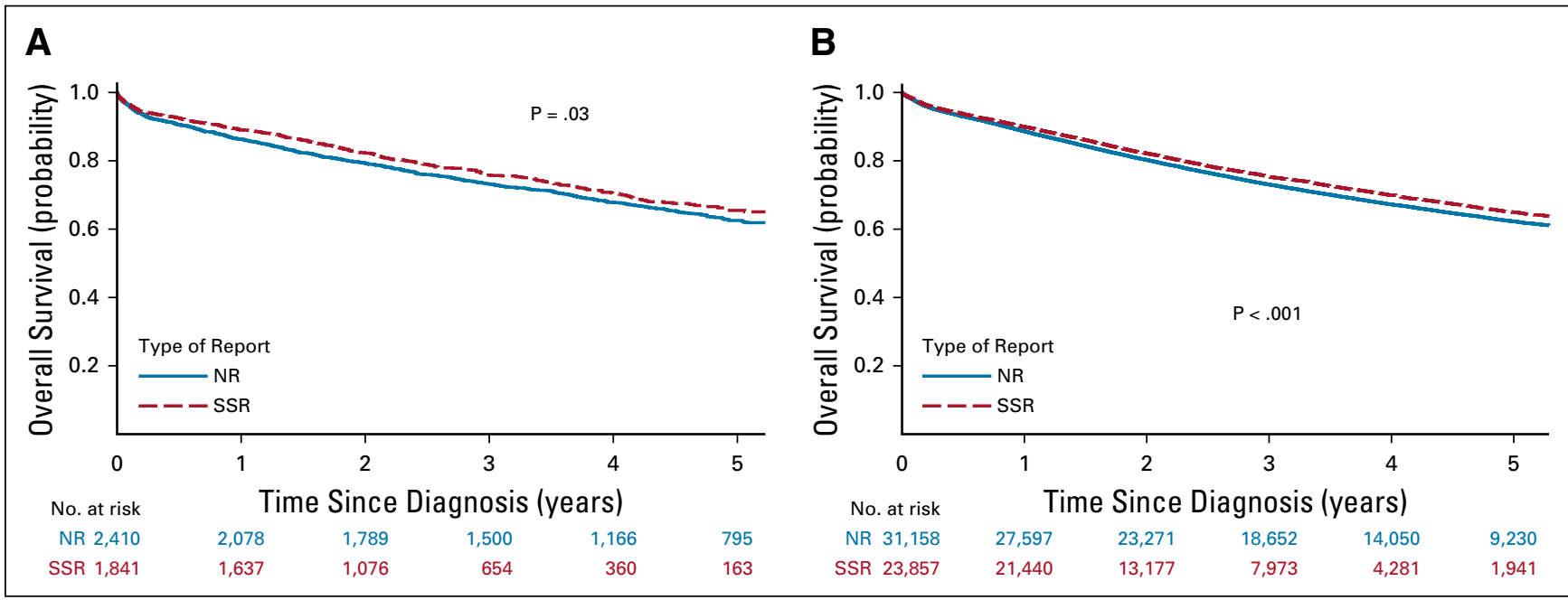

FIG 4. (A) Overall survival of Dutch patients with colorectal cancer (CRC) with high-risk stage II disease. There is a small but consistent overall survival benefit in the patients whose tumors were reported with standardized structured reporting (SSR). $P=.026$. (B) All stages CRC according to the type of pathology report. There is a small but consistent overall survival benefit in the patients whose tumors were reported with SSR. $P<.001$. NR, narrative report. 
Of more importance, we also proved that the introduction of SSR leads to better-quality pathology reporting. Increased awareness of the critical issues in pathology assessment, such as CRM in rectal cancer and numbers of LNs in CRC, began before the introduction of SSR in the Netherlands, as can be observed in Figures $2 \mathrm{C}$ and $2 \mathrm{D}$, with increased reporting since 2007 . The introduction of SSR accelerated this process, as can be observed from the rapid increase in the proportion of complete reports (Fig 3A) and the mean number of examined LNs per laboratory after they started with SSR (Fig 3B). To evaluate the improved quality of pathologic examination, the use of predefined quality indicators, such as LN count, EMVI detection remains to a certain extent artificial.

Numerous studies have examined the quality of microscopic evaluation by comparing the results of slide sets scored by different groups of pathologists ${ }^{25-28}$; however, clinical relevance is more directly investigated if the relation of a prognostic factor with outcome is evaluated. Therefore, we examined the prognostic value of the different histologic factors in both the SSR and NR settings and compared their AIC values, as is often used in comparing different staging systems. ${ }^{29}$ We were able to show that for all relevant factors the prognostic value was better when SSR was used. This suggests that increased awareness, perhaps in combination with the possibility of directly checking the definitions (provided in SSR setting), leads to better histologic evaluation.

The effect of improved communication on patient care has been evaluated in the setting of multidisciplinary team meetings. ${ }^{30}$ In general, these studies report higher rates of appropriate treatment, better adherence to clinical guidelines, and better survival. ${ }^{31-33}$ In the current study, we have

\section{AFFILIATIONS}

${ }^{1}$ Radboudumc, Nijmegen, the Netherlands

2PALGA Foundation, Houten, the Netherlands

${ }^{3}$ Dutch ColoRectal Surgical Audit, Leiden, the Netherlands

${ }^{4}$ Netherlands Comprehensive Cancer Organization, Utrecht, the Netherlands

${ }^{5}$ Symbiant Pathology Expert Centre, Alkmaar, the Netherlands

\section{CORRESPONDING AUTHOR}

Iris D. Nagtegaal, MD, PhD, Department of Pathology 824, Radboudumc, PO Box 9101, 6500 HB, Nijmegen, the Netherlands; Twitter: @radboudumc; e-mail: iris.nagtegaal@radboudumc.nl.

\section{SUPPORT}

Supported by Grant No. KWF- KUN 2013-6354 from the Dutch Cancer Society.

\section{AUTHOR CONTRIBUTIONS}

Conception and design: Caro E. Sluijter, Theo Wiggers, Henk-Jan van Slooten, Lucy I.H. Overbeek, Iris D. Nagtegaal

Administrative support: Iris D. Nagtegaal focused on these items as a measurement of the effects on patient care. We demonstrated that the administration of adjuvant chemotherapy in patients with high-risk stage Il disease was significantly higher in those with SSR. In addition, appropriate staging occurred in more patients, particularly with regard to discriminating patients with high-risk from low-risk stage II CRC. Use of SSR indirectly leads to outcome benefits, as demonstrated by the difference in overall survival between patients with SSR and NR, which remains present after adjustment for the year of diagnosis and patient and tumor characteristics. We realize that in the current setup, inherent biases are present that might reflect the higher quality of the multidisciplinary team rather than use of SSR. However, given the results from the figures that show changing trends before and after the introduction of SSR (Fig 3), this bias does not explain the whole effect.

Nevertheless, a randomized clinical trial is the most appropriate design to test the effects on patient outcomes; however, such a design is impractical under the current circumstances because of the widespread implementation of SSR in our country. Therefore, we adopted a whole population cohort study in which we controlled for potentially confounding factors, such as the type of hospital and the year of diagnosis. We therefore believe that our findings are robust.

In conclusion, the nationwide implementation of SSR for CRC resulted in increased completeness of pathology reports, higher-quality pathology evaluation, and better outcomes for patients. These findings support the widespread introduction of SSR in oncology.

Collection and assembly of data: Caro E. Sluijter, Lucy I.H. Overbeek, Iris D. Nagtegaal

Data analysis and interpretation: Caro E. Sluijter, Frans van Workum, Theo Wiggers, Carlijn van de Water, Otto Visser, Lucy I.H. Overbeek, Iris D. Nagtegaal

Manuscript writing: All authors

Final approval of manuscript: All authors

Accountable for all aspects of the work: All authors

AUTHORS' DISCLOSURES OF POTENTIAL CONFLICTS OF INTEREST The following represents disclosure information provided by authors of this manuscript. All relationships are considered compensated. Relationships are self-held unless noted. I = Immediate Family Member, Inst = My Institution. Relationships may not relate to the subject matter of this manuscript. For more information about ASCO's conflict of interest policy, please refer to www.asco.org/rwc or ascopubs.org/cci/authorcenter.

Theo Wiggers

Leadership: Incision

Stock and Other Ownership Interests: Incision

No other potential conflicts of interest were reported. 


\section{REFERENCES}

1. Gray R, Barnwell J, McConkey C, et al: Adjuvant chemotherapy versus observation in patients with colorectal cancer: A randomised study. Lancet 370:2020-2029, 2007

2. Benson AB III, Schrag D, Somerfield MR, et al: American Society of Clinical Oncology recommendations on adjuvant chemotherapy for stage II colon cancer. J Clin Oncol 22:3408-3419, 2004

3. Ellis DW, Srigley J: Does standardised structured reporting contribute to quality in diagnostic pathology? The importance of evidence-based datasets. Virchows Arch 468:51-59, 2016

4. Sluijter CE, van Lonkhuijzen LR, van Slooten $\mathrm{HJ}$, et al: The effects of implementing synoptic pathology reporting in cancer diagnosis: A systematic review. Virchows Arch 468:639-649, 2016

5. Buchwald P, Olofsson F, Lörinc E, et al: Standard protocol for assessment of colon cancer improves the quality of pathology. Colorectal Dis 13:e33-e36, 2011

6. Cross SS, Feeley KM, Angel CA: The effect of four interventions on the informational content of histopathology reports of resected colorectal carcinomas. J Clin Pathol 51:481-482, 1998

7. Porter GA, Urquhart RL, Rheaume D, et al: Clinical information available to oncologists in surgically treated rectal cancer: Room to improve. Curr Oncol 20:166-172, 2013

8. Ihnát P, Delongová P, Horáček J, et al: The impact of standard protocol implementation on the quality of colorectal cancer pathology reporting. World J Surg 39:259-265, 2015

9. Siriwardana PN, Pathmeswaran A, Hewavisenthi J, et al: Histopathology reporting in colorectal cancer: A proforma improves quality. Colorectal Dis 11:849-853, 2009

10. Casati B, Bjugn R: Structured electronic template for histopathology reporting on colorectal carcinoma resections: Five-year follow-up shows sustainable longterm quality improvement. Arch Pathol Lab Med 136:652-656, 2012

11. Messenger DE, McLeod RS, Kirsch R: What impact has the introduction of a synoptic report for rectal cancer had on reporting outcomes for specialist gastrointestinal and nongastrointestinal pathologists? Arch Pathol Lab Med 135:1471-1475, 2011

12. Chan NG, Duggal A, Weir MM, et al: Pathological reporting of colorectal cancer specimens: A retrospective survey in an academic Canadian pathology department. Can J Surg 51:284-288, 2008

13. Beattie GC, McAdam TK, Elliott S, et al: Improvement in quality of colorectal cancer pathology reporting with a standardized proforma: A comparative study. Colorectal Dis 5:558-562, 2003

14. Integraal Kankercentrum Nederland: Dutch guidelines for colorectal cancer. http://www.oncoline.nl/colorectaalcarcinoom

15. Schouten LJ, Höppener P, van den Brandt PA, et al: Completeness of cancer registration in Limburg, the Netherlands. Int J Epidemiol 22:369-376, 1993

16. Casparie M, Tiebosch AT, Burger G, et al: Pathology databanking and biobanking in the Netherlands, a central role for PALGA, the nationwide histopathology and cytopathology data network and archive. Cell Oncol 29:19-24, 2007

17. Kolfschoten NE, Marang van de Mheen PJ, Gooiker GA, et al: Variation in case-mix between hospitals treating colorectal cancer patients in the Netherlands. Eur J Surg Oncol 37:956-963, 2011

18. World Health Organization: International Classification of Diseases for Oncology. http://codes.iarc.fr/home

19. Messenger DE, Driman DK, McLeod RS, et al: Current practice patterns among pathologists in the assessment of venous invasion in colorectal cancer. J Clin Pathol 64:983-989, 2011

20. Cook DA, Coory M, Webster RA: Exponentially weighted moving average charts to compare observed and expected values for monitoring risk-adjusted hospital indicators. BMJ Qual Saf 20:469-474, 2011

21. Burnham KP, Anderson DR: Model Selection and Multimodel Interference: A Practical Information-Theoretic Approach (ed 2). New York, NY, Springer, 2002

22. Idowu MO, Bekeris LG, Raab S, et al: Adequacy of surgical pathology reporting of cancer: A College of American Pathologists Q-Probes study of 86 institutions. Arch Pathol Lab Med 134:969-974, 2010

23. Rigby K, Brown SR, Lakin G, et al: The use of a proforma improves colorectal cancer pathology reporting. Ann R Coll Surg Engl 81:401-403, 1999

24. Srigley J, Lankshear S, Brierley J, et al: Closing the quality loop: Facilitating improvement in oncology practice through timely access to clinical performance indicators. J Oncol Pract 9:e255-e261, 2013

25. Kojima M, Puppa G, Kirsch R, et al: Blood and lymphatic vessel invasion in pT1 colorectal cancer: An international concordance study. J Clin Pathol 68:628-632, 2015

26. Koelzer VH, Zlobec I, Berger MD, et al: Tumor budding in colorectal cancer revisited: Results of a multicenter interobserver study. Virchows Arch 466:485-493, 2015

27. Kirsch R, Messenger DE, Riddell RH, et al: Venous invasion in colorectal cancer: Impact of an elastin stain on detection and interobserver agreement among gastrointestinal and nongastrointestinal pathologists. Am J Surg Pathol 37:200-210, 2013

28. Ueno H, Mochizuki H, Shirouzu K, et al: Multicenter study for optimal categorization of extramural tumor deposits for colorectal cancer staging. Ann Surg 255:739-746, 2012

29. Nagtegaal ID, Tot T, Jayne DG, et al: Lymph nodes, tumor deposits, and TNM: Are we getting better? J Clin Oncol 29:2487-2492, 2011

30. Pillay B, Wootten AC, Crowe $\mathrm{H}$, et al: The impact of multidisciplinary team meetings on patient assessment, management and outcomes in oncology settings: A systematic review of the literature. Cancer Treat Rev 42:56-72, 2016

31. MacDermid E, Hooton G, MacDonald M, et al: Improving patient survival with the colorectal cancer multi-disciplinary team. Colorectal Dis 11:291-295, 2009

32. Wille-Jørgensen P, Sparre P, Glenthøj A, et al: Result of the implementation of multidisciplinary teams in rectal cancer. Colorectal Dis 15:410-413, 2013

33. Palmer G, Martling A, Cedermark B, et al: Preoperative tumour staging with multidisciplinary team assessment improves the outcome in locally advanced primary rectal cancer. Colorectal Dis 13:1361-1369, 2011 


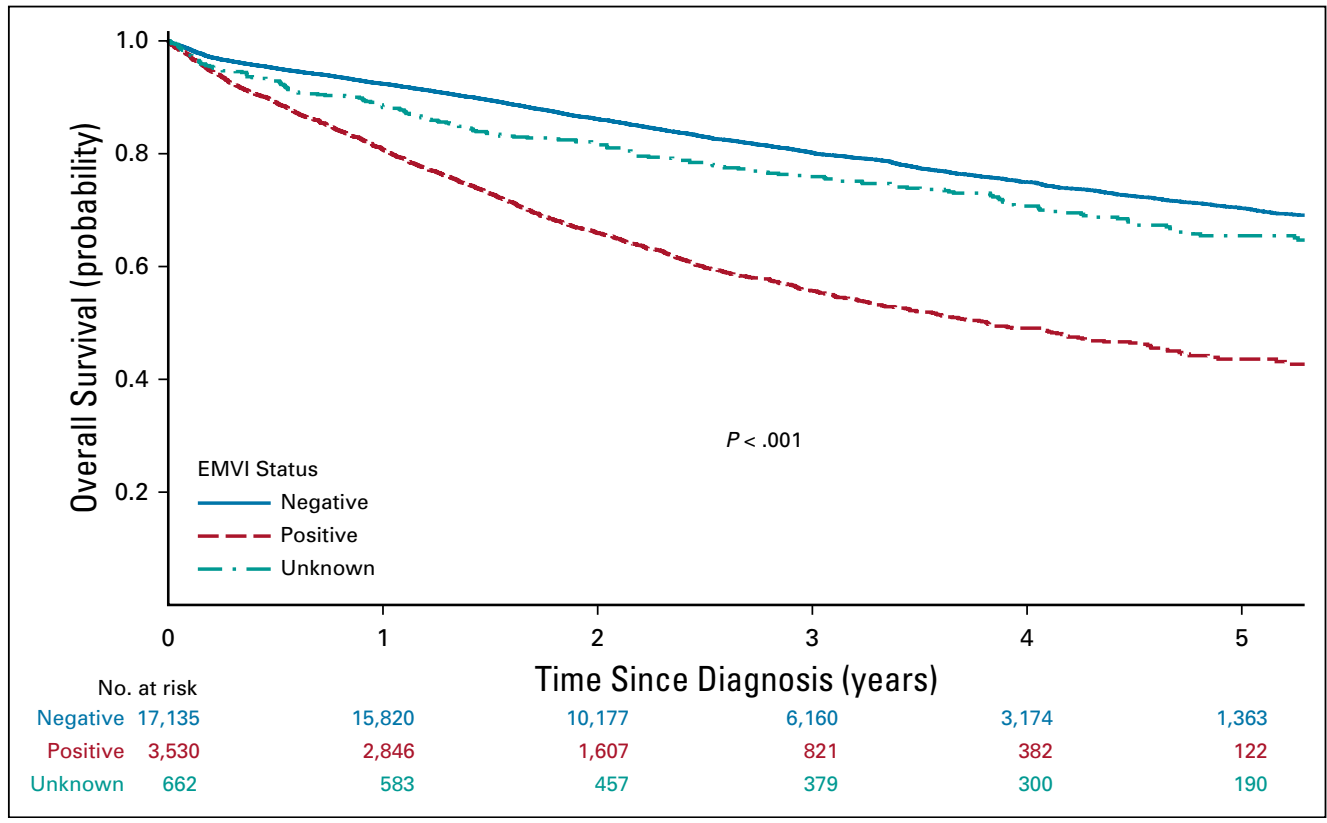

FIG A1. Survival curves to study the impact of positive, negative, and unknown extramural vascular invasion for standardized structured reporting.

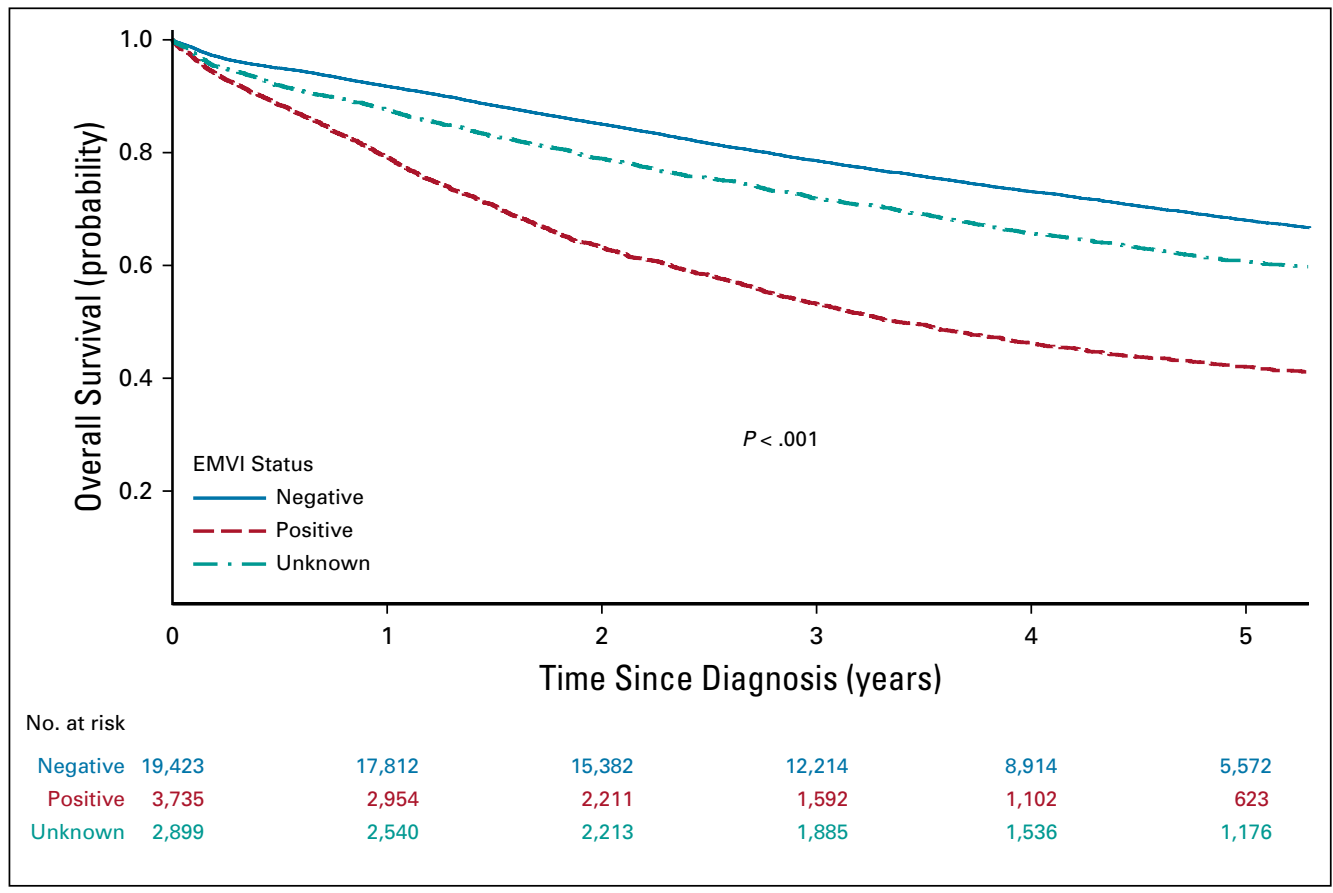

FIG A2. Survival curves to study the impact of positive, negative, and unknown extramural vascular invasion for standardized structured reporting and narrative report. 


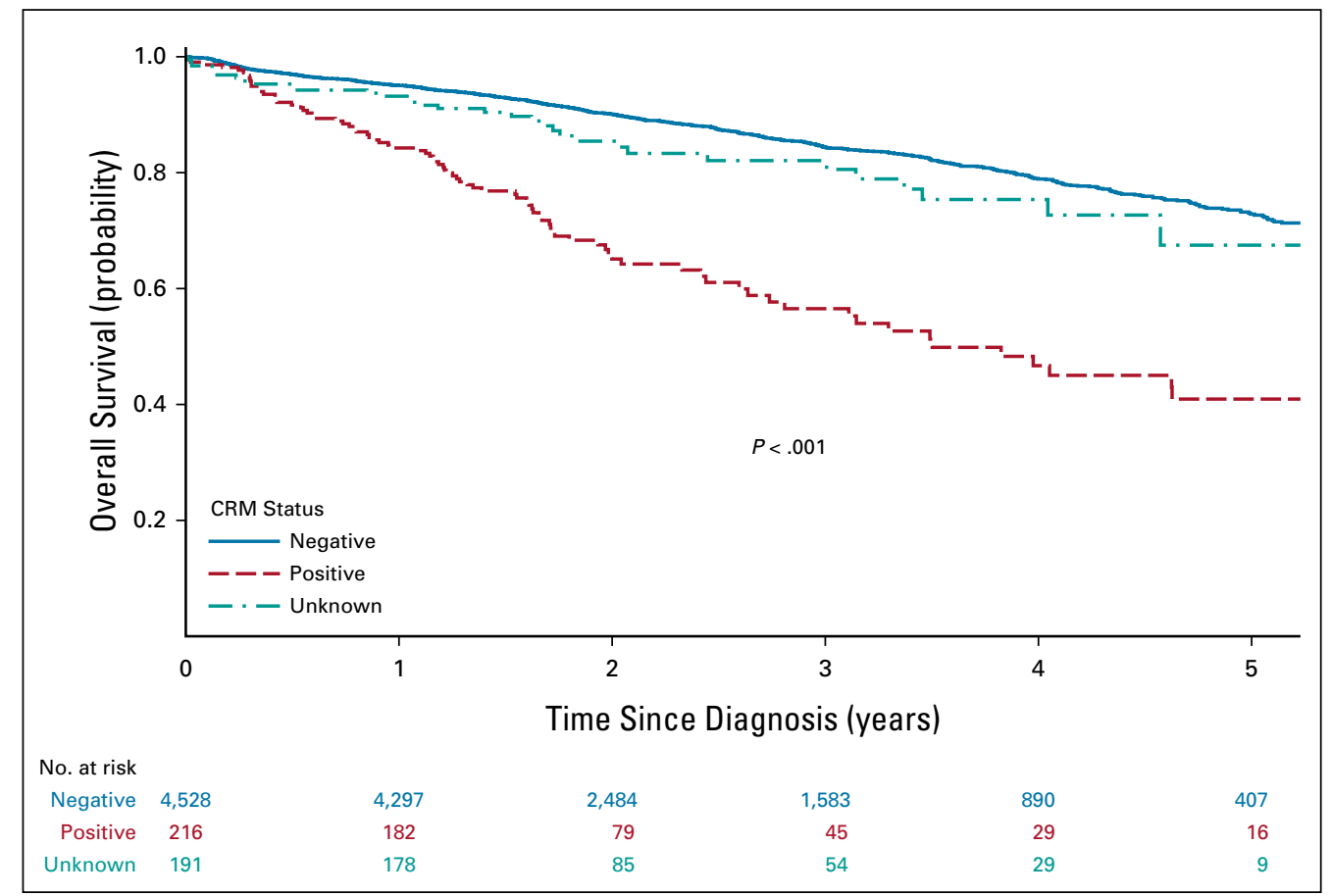

FIG A3. Survival curves for the impact of circumferential resection margin involvement for rectal carcinoma for standardized structured reporting.

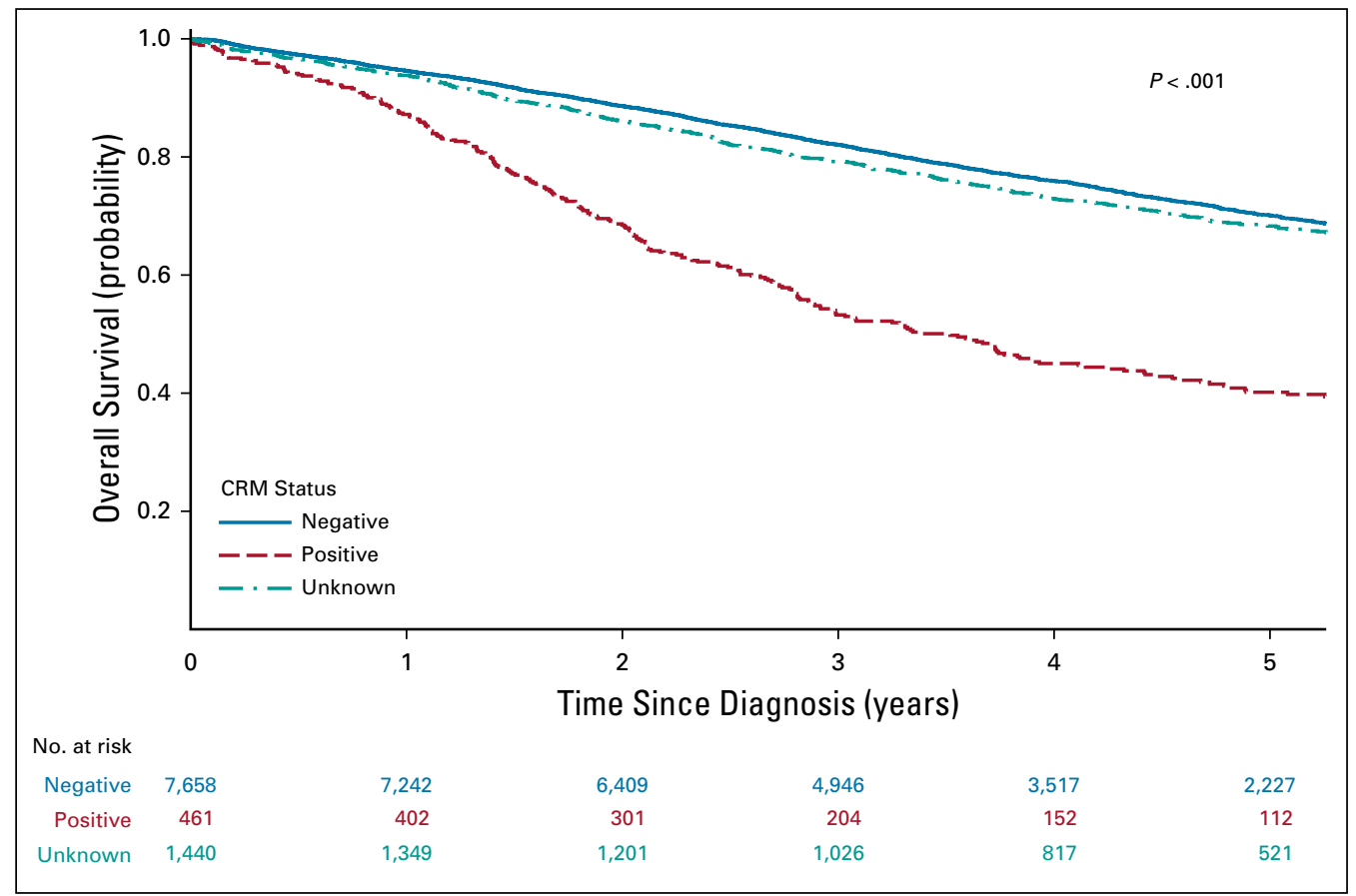

FIG A4. Survival curves for the impact of circumferential resection margin involvement for rectal carcinoma for narrative report.

10 (C) 2019 by American Society of Clinical Oncology 


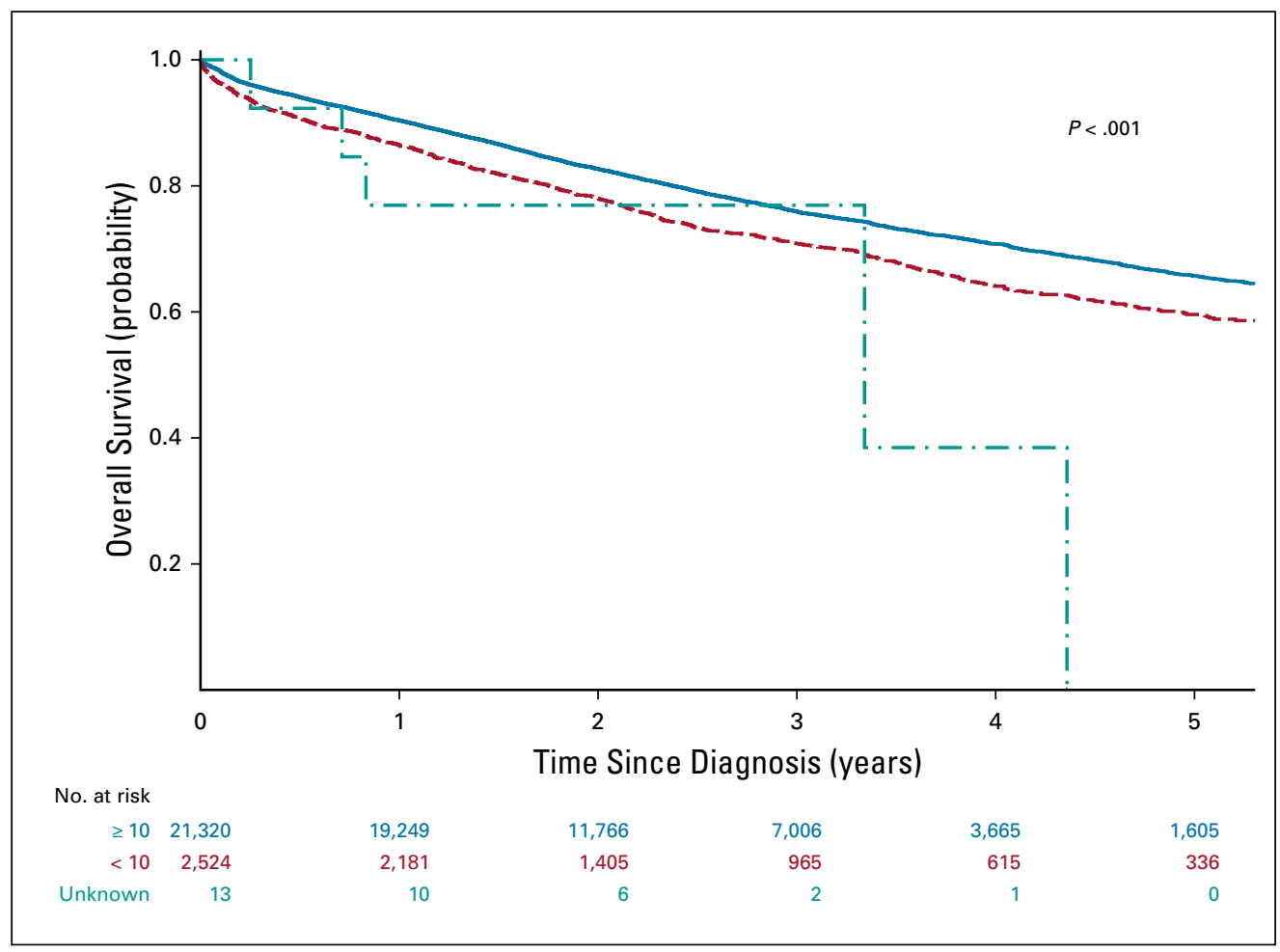

FIG A5. Survival curves for the impact of 10 or more examined lymph nodes for standardized structured reporting.

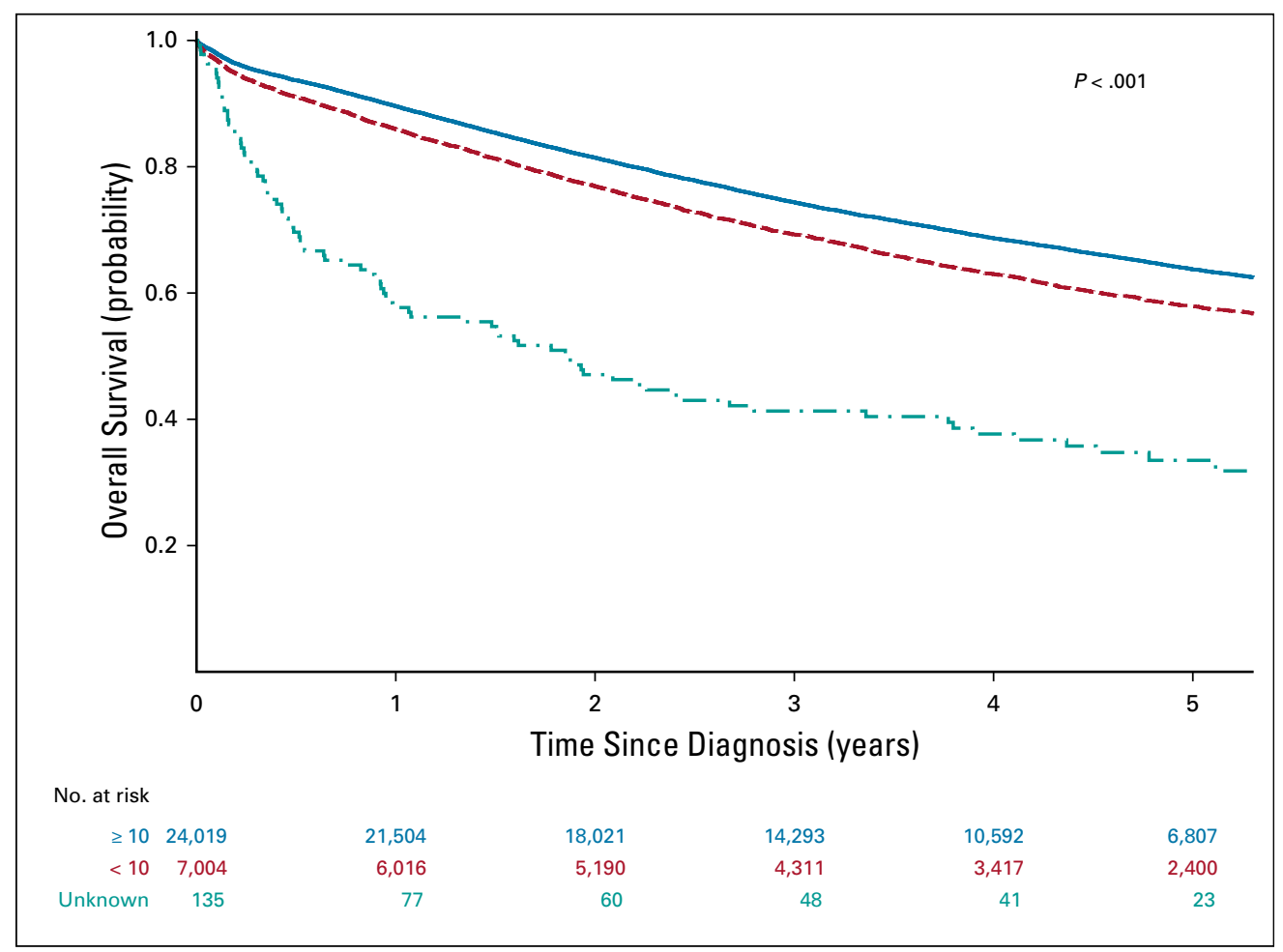

FIG A6. Survival curves for the impact of 10 or more examined lymph nodes for narrative report. 
TABLE A1. Effects of SSR on Completeness and Quality Indicators

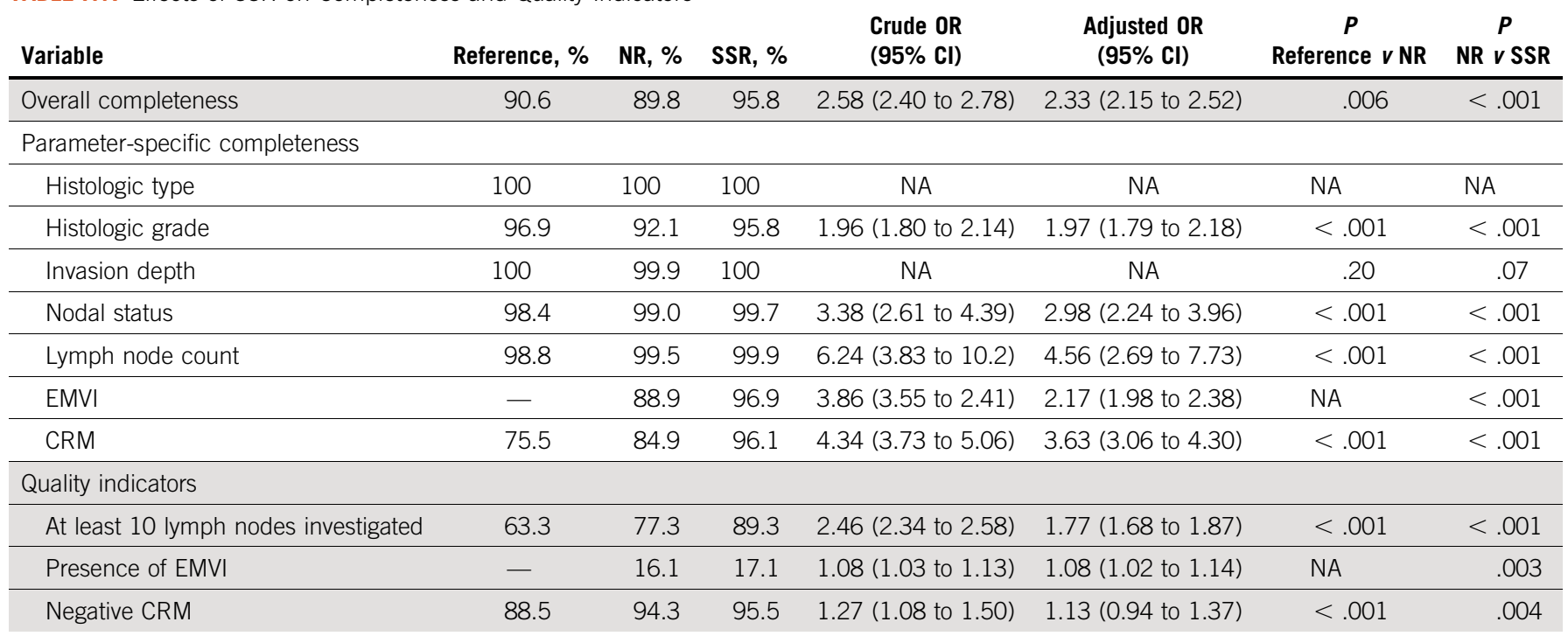

NOTE. Adjusted OR is corrected for year of diagnosis.

Abbreviations: CRM, circumferential margin; EMVI, extramural vascular invasion; NA, not assessable; NR, narrative reporting; OR, odds ratio; SSR, standardized structured reporting. 